\title{
MedienPädagogik
}

Zeitschrift für Theorie und Praxis der Medienbildung

\section{Lernen mit und über digitale Medien im Sachunterricht}

\section{Entwicklung eines vielperspektivischen Konzepts zur Erschliessung digitaler Medien}

Carmen Kunkel und Markus Peschel

\section{Zusammenfassung}

Das Lernen mit und über digitale Medien muss Kindern bereits in der Grundschule ermöglicht werden, um ihnen frühzeitig eine kritische und konstruktive Auseinandersetzung mit digitalen Medien aus bildungsorientierter Perspektive zu ermöglichen. Aufgrund seiner welterschliessenden Kernaufgabe und seines vielperspektivischen Prinzips ist der Sachunterricht das zentrale Unterrichtsfach in der Grundschule für das Lernen mit und über digitale Medien im Sinne einer schulischen Medienbildung. Ausgehend von ihrer Lebenswelt sollen Grundschülerinnen und -schüler die Möglichkeit erhalten, digitale Medien als Sache des Sachunterrichts in ihrer Komplexität zu erschliessen. Dafür ist es notwendig, Konzepte für das Lernen über digitale Medien in einem vielperspektivischen Sachunterricht zu entwickeln, in denen zu prüfen ist, unter welchen Bedingungen digitale Technik für ein Lernen mit digitalen Medien sinnvoll eingesetzt werden kann. Mit dieser Herausforderung haben sich Studierenden des Lehramts Primarstufe an der Universität des Saarlandes im Sommersemester 2019 beschäftigt. Der Artikel stellt die Ergebnisse der seminar-begleitenden Konzeptentwicklung vor und gibt Ausblicke, wie diese ausgebaut werden können. 


\title{
Learning with and about ICT in primary education
}

\begin{abstract}
Learning with and about ICT must be accessed to children on from primary education in order to allow them to establish a critical-constructive examination of ICT in an educational understanding. As it is the core task of primary social and science education to enable students to self-construct and self-explore their environment in a multiperspective manner, primary social and science education functions as an essential subject in primary school to address learning with and about ICT in accordance to media literacy. Originating from the students' living environment, they must be given the possibility to open up ICT/digital media as a complex matter of primary social and science education. Therefore, concepts on learning with and about ICT in multiperspective social and science primary education must be developed and then be examined regarding the conditions that allow for a learning with and about ICT by reasonable deployment of digital techniques. In summer 2019, several university students in teacher education encountered this challenge. The presented article outlines the development stages and outcomes of such concepts that were designed during an educational seminar. It then offers an outlook towards possible further advancements.
\end{abstract}

\section{Digitale Medien in der Lebenswelt von Kindern}

Kinder wachsen zunehmend in einem digitalen Zeitalter auf (Mitzlaff und Speck-Hamdan 1998, 8; Moser 2019, 96). In Spielsachen finden sich in smart toys und programmierbaren Robotern Informatiksysteme (Gesellschaft für Informatik 2019, V). Die LeYo!-Kinderbuch-Reihe von Carlsen (2019) nutzt wie LEGO Playgrounds (LEGO 2018) Augmented Reality (AR) und am Markt existieren zahlreiche Spiele-Apps für jedes Kindesalter. Technologie und Digitalisierung haben sich nach Angaben der Spielwarenmesse vom Trend zur festen Grösse im Bereich Spielwaren entwickelt (Spielwarenmesse 2019). Peter Thomas fasst zusammen: «Elektronisches Spielzeug macht 2019 deutlich, dass Spielen heute so intelligent und umfassend von smarter Technologie unterstützt wird wie nie zuvor» (Thomas 2019). Kinder haben im privaten Umfeld Zugriff auf verschiedene digitale Medien wie 
Smartphones, Laptops, Tablets, Computer und in nahezu allen Haushalten mit sechs- bis dreizehnjährigen Kindern existiert ein Zugang zum Internet (Medienpädagogischer Forschungsverbund Südwest 2018, 31; 2019, 1). Diese digitalen Medien werden von Kindern zum Spielen, aber auch bereits zur Nutzung von Online-Diensten wie Suchmaschinen, YouTube, WhatsApp und Social Media- Angeboten wie Snapchat, Instagram, Facebook und TikTok verwendet (Medienpädagogischer Forschungsverbund Südwest 2019). Während die Digitalisierung ${ }^{1}$ und Mediatisierung zunehmend die Lebenswelt von Kindern durchdringt (Grundschulverband 2015; Kultusministerkonferenz 2016, 11; Gesellschaft für Informatik 2016, 2; 2019, V; AG Medien \& Digitalisierung der GDSU 2019, 1), bleiben die Funktions- und Arbeitsweisen digitaler Technik (Gervé 2015, 497; Gesellschaft für Informatik 2019, V) sowie deren Einfluss auf die eigene Persönlichkeitsentwicklung (Grundschulverband 2018, 1) für die jungen Nutzenden meist undurchsichtig und unverständlich. Die alleinige (unterhaltungsorientierte) Nutzung kann folglich die digitale Naivität (Döbeli Honegger 2007) der Kinder nicht überwinden (Irion 2018, 5; AG Medien \& Digitalisierung der GDSU 2019, 6; Lenkungskreis der Initiative KBoM 2019).

Kinder müssen somit im Umgang mit digitalen Medien begleitet werden und benötigen Unterstützung aus bildungsorientierter Perspektive (AG Medien \& Digitalisierung der GDSU 2019, 3). Daher ist es Aufgabe der Schule, das Lernen mit und über digitale Medien im Sinne einer schulischen Medienbildung (Irion und Peschel 2016, 13) in den Unterricht zu integrieren (Klafki 1992; Döbeli Honegger 2016, 76f.; Irion 2018; Gesellschaft für Informatik 2019). Empfehlungen aus den Fachdidaktiken (Gesellschaft für Informatik 2019; AG Medien \& Digitalisierung der GDSU 2019), der Medienpädagogik (Sektion Medienpädagogik, DGfE 2016; Lenkungskreis der Initiative KBoM 2019), dem Grundschulverband (Grundschulverband 2015; 2018) und den Kultusministerkonferenzen (Kultusministerkonferenz 2012; 2016) bestätigen, dass das Lernen mit und über digitale Medien frühzeitig beginnen muss. Als erste verbindliche Pflichtschule für alle Kinder (Peschel 2019a, 28) legt die Grundschule daher die Basis im Umgang mit digitalen

1 Hier verstanden als Technik der Darstellung, Verarbeitung, Speicherung und Verbreitung von numerischen Grössen, Text, Bild, Ton und Film in Zahlencodes mit tiefgreifenden, gesellschaftlichen Implikationen (AG Medien \& Digitalisierung der GDSU 2019, 3). 
Medien (Irion 2018, 7) und ermöglicht in einem dauerhaften, pädagogisch strukturierten und begleiteten Prozess allen Schülerinnen und Schülern eine konstruktive und kritische Auseinandersetzung mit der Medienwelt (Kultusministerkonferenz 2012, 3). Wie dies konkret umzusetzen ist, wird vielfach diskutiert ${ }^{2}$, denn momentan ist das Lernen mit und über digitale Medien noch kein fester Bestandteil des Unterrichts an deutschen Grundschulen (Gervé 2015; Eickelmann 2016; Behrens u. a. 2017; Thom u. a. 2017; Medienpädagogischer Forschungsverbund Südwest 2018), in denen digitale Medien nur sehr eingeschränkt eingesetzt werden (Behrens u. a. 2017, 29; Thom u. a. 2017). Es ist aktuell notwendig, durch den Abbau bewahrpädagogischer Haltungen (Peschel 2019a, 37) und den Aufbau entsprechender Lernangebote den Fachunterricht in Grundschulen so umzusetzen, dass digitale Kompetenzen fachlich und fachliche Kompetenzen digital gefördert werden (Gesellschaft für Fachdidaktik 2018).

Grundschulen stehen bei dieser Aufgabe vor vielvielfältigen und teilweise noch ungeklärten Herausforderungen (Peschel 2019b; Gesellschaft für Medienpädagogik und Kommunikationskultur 2019). Aufgrund der Tatsache, dass der Bildungserfolg in einer zunehmend durch Digitalisierung und Mediatisierung geprägten Welt mit der sozioökonomischen Lage der Schülerfamilien gekoppelt ist (Eickelmann, Bos, und Labusch 2019, 27; Reiss u. a. 2019, 10f.), besteht dringender Handlungsbedarf. Grundschulen benötigen ein auf ihre Bedürfnisse angepasstes Medienkonzept. Weil die Auswahl einer bestimmten technischen Schulausstattung aus fachlichen, fachdidaktischen und medienpädagogischen Überlegungen resultiert (Gewerkschaft Erziehung und Wissenschaft 2019), müssen die Schulen in diesem Prozess unterstützt und letztlich auch beraten werden, welche (digitalen) Medien sich zur Umsetzung der Ziele eignen. Als Voraussetzung für die Integration ausgewählter digitaler Medien, ist eine ausreichend schnelle Anbindung an das Internet in allen Klassenzimmern notwendig (Grundschulverband 2018). Für den Einsatz im Unterricht eignen sich benutzungsfreundliche und altersgerechte Hard- und Softwarelösungen, deren Wartung und Support nicht von Lehrkräften, sondern durch

2 Vgl. Kultusministerkonferenz 2012; 2016; Döbeli Honegger 2016; Gesellschaft für Informatik 2016; Gesellschaft für Fachdidaktik 2018; Gesellschaft für Informatik 2019; AG Medien \& Digitalisierung der GDSU 2019; Lenkungskreis der Initiative KBoM 2019; Tulodziecki, Grafe, und Herzig 2019. 
ausgebildetes Personal gewährleistet werden muss (Grundschulverband 2018; Peschel 2019b; AG Medien \& Digitalisierung der GDSU 2019). Um Kindern das Lernen mit und über digitale Medien zu ermöglichen, benötigen Lehrkräfte eine wissenschaftliche und praxisnahe Aus- und Fortbildung (Grundschulverband 2018; Peschel 2019b; AG Medien \& Digitalisierung der GDSU 2019; Gesellschaft für Medienpädagogik und Kommunikationskultur 2019), da diese als keystone species (Davis, Eickelmann, und Zaka 2013, 439) die Qualität und die Umsetzung einer zeitgemässen, grundschulgerechten Bildung für eine zunehmend durch digitale Medien beeinflusste Welt (Eickelmann, Bos, und Labusch 2019, 27f.) erheblich bedingen (Drossel u. a. 2019, 205; Gesellschaft für Medienpädagogik und Kommunikationskultur 2019; Behrens u. a. 2017; Thom u. a. 2017). Die Umsetzung der genannten Herausforderungen setzt eine entsprechend zukunftsorientierte Etatplanung voraus, um dem Bedarf von Grundschulen nachzukommen (Grundschulverband 2018; Peschel 2019b), welche die Hälfte aller Allgemeinbildenden Schulen bilden (Statistisches Bundesamt 2019).

Dass die digitalisierungsbezogenen Ansätze innerhalb von Grundschulen noch nicht ausreichen, wird aus den aktuellen Ergebnissen der ICILS2018-Studie zur Medienkompetenz von Schülerinnen und Schülern deutlich. Nur etwas mehr als ein Drittel der Schülerinnen und Schüler der achten Klasse verfügen über mindestens fünf Jahre schulische Erfahrungen im Umgang mit Computern oder Notebooks bzw. Laptops (Schaumburg u. a. 2019, 267), konnten also bis zum Ende der dritten Klasse oder früher bildungsorientierte Erfahrungen mit digitalen Medien sammeln. Im internationalen Vergleich weisen bis auf Kasachstan und Italien alle anderen ICILS-2018-Teilnehmerländer sowie die Vergleichsgruppe internationaler Mittelwert und die Vergleichsgruppe EU signifikant höhere Anteile auf als Deutschland (Schaumburg u. a. 2019, 257). Weil die Dauer der Erfahrung mit digitalen Medien signifikant positiv mit den computer- und informationsbezogenen Kompetenzen der getesteten Schülerinnen und Schüler zusammenhängt (Schaumburg u. a. 2019, 267), gibt dies Hinweise darauf, dass die in ICILS betrachteten Kompetenzen über einen langjährigen Zeitraum zu entwickeln sind und Förderpotentiale im Grundschulbereich liegen (Schaumburg u. a. 2019, 267). Diese müssen dringend genutzt werden, denn ein Drittel der getesteten Schülerinnen und Schüler verfügt nur 
über sehr rudimentäre und basale computer- und informationsbezogene Kompetenzen auf dem Niveau der unteren beiden Kompetenzstufen. Im internationalen Vergleich ist eine Leistungsspitze in Deutschland kaum vorhanden (Eickelmann, Bos, und Labusch 2019, 27f.). Weiterhin konnten in zahlreichen Bereichen wie dem mittleren und unteren Leistungsniveau der computer- und informationsbezogenen Kompetenzen der Schülerinnen und Schüler in Deutschland keine signifikanten Unterschiede im Vergleich zu 2013 festgestellt werden (Eickelmann, Bos, und Labusch 2019, 13).

Für eine erfolgreiche Förderung von Medienkompetenzen ist nicht allein die Quantität eingesetzter digitaler Medien ausschlaggebend (Drossel u. a. 2019, 233; Grundschulverband 2018). Entscheidend für den Erfolg ist die Qualität des didaktisch geplanten Fachunterrichts unter Einbezug von digitalen Medien. Demnach werden digitale Medien anhand abgeleiteter fachlicher, fachdidaktischer und medienpädagogischer Lernziele reflektiert in den jeweiligen Fachunterricht eingebunden (Mitzlaff 2016, 22; Peschel 2016, 7f.; Gesellschaft für Fachdidaktik 2018), um Schülerinnen und Schüler sowohl ein Lernen mit, als auch ein Lernen über digitale $\mathrm{Me-}$ dien zu ermöglichen. In der Grundschule ist der Sachunterricht das zentrale Fach für das Lernen mit und über digitale Medien (Gervé 2019), um Schülerinnen und Schüler in die Lage zu versetzen, eine zunehmend durch Digitalisierung und Mediatisierung geprägte Lebenswelt kritisch zu reflektieren, zu verstehen und mitgestalten zu können ${ }^{3}$ (Köhnlein 2012, 28; Gervé 2015, 496; Schorb, Hartung-Griemberg, und Dallmann 2017, 135; AG Medien \& Digitalisierung der GDSU 2019, 2). Die Erschliessung digitaler Medien durch das Lernen mit und über digitale Medien stellt den Sachunterricht vor Herausforderungen: Es müssen Konzepte für das Lernen über digitale Medien entwickelt werden (AG Medien \& Digitalisierung der GDSU 2019, 6), in denen zu prüfen ist, unter welchen Bedingung digitale Technik für das Lernen mit digitalen Medien sinnvoll eingesetzt werden kann (AG Medien \& Digitalisierung der GDSU 2019, 1). Mit dieser Herausforderung haben sich Studierende des Lehramts Primarstufe an der Universität des Saarlandes im Sommersemester 2019 beschäftigt. Ziel war die Prüfung,

3 Bereits 1992 formulierte Wolfgang Klafki die Auseinandersetzung mit Gefahren und Möglichkeiten der neuen technischen Steuerungs-, Informationsund Kommunikationsmedien als eine zentrale Dimension des Sachunterrichts (Klafki 1992, 21). 
welche Lerninhalte über digitale Medien aus sachunterrichtsdidaktischer Sicht sinnvoll im Unterricht zu entwickeln sind und wie sich Einsatz und Auswahl digitaler Medien im Sachunterricht begründen. Die Entwicklungsschritte und Ergebnisse werden im Folgenden dargestellt.

\section{Digitale Medien als Sache des Sachunterrichts}

Ausgangspunkt des Sachunterrichts ist die Lebenswelt der Kinder. Seine Aufgabe ist es Schülerinnen und Schüler darin zu unterstützen, diese [...] sachbezogen zu verstehen, sie sich auf dieser Grundlage bildungswirksam $\mathrm{zu}$ erschliessen und sich darin zu orientieren, mitzuwirken und zu handeln (GDSU 2013, 9). Digitale Medien sind, wie bereits dargestellt, Teil der kindlichen Lebenswelt. Ihre Erschliessung begründet daher das Lernen mit und über digitale Medien als Sache des Sachunterrichts (Gervé 2016). Das Lernen über digitale Medien als Erscheinungsformen der Digitalisierung, erfordert in Anbetracht ihrer Komplexität eine umfassende Betrachtung aus verschiedenen Perspektiven (Gesellschaft für Informatik 2016, 2; AG Medien \& Digitalisierung der GDSU 2019, 5), die aufgrund gegenseitiger Wechselwirkungen aufeinander bezogen (Gesellschaft für Informatik 2016, 2) beziehungsweise miteinander vernetzt werden müssen (AG Medien \& Digitalisierung der GDSU 2019, 5). Das vielperspektivische Prinzip ist charakteristisch für den Sachunterricht (Köhnlein 2011; Köhnlein, Marquardt-Mau, und Duncker 2013; GDSU 2013; Kahlert 2016; Lauterbach 2017; Giest, Hartinger, und Tänzer 2017). Aus diesem Grund eignet sich der Sachunterricht für Grundschulkinder in besonderer Weise zur Erschiessung digitaler Medien. Vielperspektivität beschreibt im Sachunterricht ein Prinzip der Vielfalt aufeinander bezogener Inhalte, Betrachtungsweisen und Methoden (Köhnlein 2012, 149) und begründet sich als didaktisches Prinzip erkenntnistheoretisch-konstruktivistisch, kognitionspsychologisch, sozialisationstheoretisch, philosophisch und anthropologisch (Köhnlein, Marquardt-Mau, und Duncker 2013, 3; Albers 2017, 12). Durch den Zugang zu den Sachen, Wissensbeständen, Wissensformen sowie Denk- Arbeits- und Handlungsweisen aus unterschiedlichen Perspektiven des Welterkennens- und -verstehens (Giest, Hartinger, und Tänzer 2017, 9f.) soll Kindern die Erschliessung von komplexen Phänomenen wie digitale 
Medien ermöglicht werden. Der Sachunterricht nimmt die «Erfahrungen und ursprünglichen Ansätze der Wirklichkeitserkundung der Kinder auf» und führt diese weiter «zu gesicherten Formen des Wissens und Könnens» (Köhnlein 2011, 10f.; GDSU 2013, 9f.). Er bezieht die kindliche/lebensweltliche und wissenschaftliche/fachliche Perspektive aufeinander (Giest, Hartinger, und Tänzer 2017, 10), nutzt dabei das Erklärungswissen der Wissenschaften und schafft Anschlussfähigkeit an spätere Fachperspektiven (Köhnlein 2011, 10f.; GDSU 2013, 9f.).

\subsection{Lernen über Medien als perspektivenvernetzender Themenbereich}

Der Sachunterricht greift im Perspektivrahmen (PR) der GDSU (2013) die Erschliessung analoger und digitaler Medien als Teil der Lebenswelt von Grundschülerinnen und -schülern in einem speziellen perspektivenvernetzenden Themenbereich (PVT) Medien auf (GDSU 2013, 83f.). Dies verdeutlicht den Stellenwert von Medien in der Lebenswelt der Kinder (Peschel 2016, 12) und ermöglicht das Lernen über Medien durch eine vertiefte Auseinandersetzung innerhalb der Perspektiven des Sachunterrichts und durch deren Vernetzung (GDSU 2013, 13; 2013, 83f;; Peschel 2016, 12). Im Perspektivrahmen des Sachunterrichts wird beschrieben, dass der Themenbereich $\mathrm{Me}$ dien vielfältige Aspekte beinhaltet, die in verschiedene Perspektiven und perspektivenübergreifende Bereiche des Sachunterrichts reichen bzw. aus denen heraus das Thema Medien aufgegriffen und bearbeitet werden kann (GDSU 2013, 84). Als vordergründige Themenfelder benennt der PR den Medienalltag, verschiedene Kommunikationsmittel und ihre Auswirkungen auf Gesellschaft und Politik, Medien als Informationsträger, Sicherheit im Netz und technische Funktionsweisen neuer Medien (GDSU 2013, 83f.). Die fünf Themenfelder werden innerhalb des Perspektivrahmens nicht näher beschrieben, weshalb unklar bleibt, welche konkreten Inhalte über digitale Medien innerhalb der bestehenden Themenbereiche der sozialwissenschaftlichen, naturwissenschaftlichen, geographischen, historischen und technischen Perspektive des Sachunterrichts erschlossen und wie diese miteinander vernetzt werden können. Die Erschliessung von perspektivenbezogenen Inhalten der Themenbereiche ist weiterhin immer in Zusammenhang mit 
den perspektivenbezogenen Denk-, Arbeits- und Handlungsweisen (DAH) der jeweiligen Perspektive zu verstehen (GDSU 2013, 12). Im Perspektivrahmen wird nicht näher beschrieben, wie die perspektivenbezogenen DAH digital gestützt als Lernen mit digitalen Medien ermöglicht werden können. In Erweiterung des Perspektivrahmens wäre es zudem denkbar, auch die perspektivenübergreifenden Denk-, Arbeits- und Handlungsweisen erkennen/verstehen, eigenständig erarbeiten, evaluieren/reflektieren, kommunizieren/zusammenarbeiten, den Sachen interessiert begegnen und umsetzen/handeln (GDSU 2013, 13) als Lernen mit digitalen Medien zu verstehen (Peschel 2016, 12). Diese sind in Ansätzen, wie beispielsweise der Einsatz von Wikis und Suchmaschinen zur eigenständigen Informationssuche, bereits im PVT Medien formuliert (GDSU 2013, 85). Ausgehend von ihren eigenen Erfahrungen sollen Schülerinnen und Schüler durch das Lernen mit und über digitale Medien im Sachunterricht die Möglichkeit erhalten, Risiken, Potentiale und Folgen digitaler Technik zu erschliessen (Irion 2018; AG Medien \& Digitalisierung der GDSU 2019) und relevante Aspekte wie beispielsweise Automatisierung, Robotik, Big Data, Virtualität, Datenspeicherung und Daten-schutz, aber auch Veränderungen in Politik, Ökonomie, Ökologie, Gesundheit und Nachhaltigkeit aufgreifen (AG Medien \& Digitalisierung der GDSU 2019, 3). Durch das Lernen mit und über digitale Medien im PVT fliessen informatische Aspekte in die Perspektiven des Sachunterrichts ein (GDSU 2013; Straube u. a. 2018; AG Medien \& Digitalisierung der GDSU 2019). Empfehlungen, welche informatischen Kompetenzen von Schülerinnen und Schülern im Primarbereich gefördert werden können, wurden seitens der Gesellschaft für Informatik vorgeschlagen (2019). Diese könnten unter Einhaltung des vielperspektivischen Prinzips im Gesamtkontext eines welterschliessenden Sachunterrichts aufgegriffen und entwickelt werden (AG Medien \& Digitalisierung der GDSU 2019). In Bezug auf das Lernen mit und über digitale Medien wurden die Inhalte der Perspektiven, der perspektivenbezogenen sowie der perspektivenübergreifenden DAH bislang noch nicht konkret fixiert. Unter Berücksichtigung der grundlegenden Struktur des Perspektivrahmens Sachunterricht, wurde dies von den Studierenden erarbeitet. 


\subsection{Lernen mit und über digitale Medien in den verschiedenen Perspektiven}

Der Perspektivrahmen der Gesellschaft für Didaktik des Sachunterrichts benennt die sozialwissenschaftliche, naturwissenschaftliche, geographische, historische und technische Perspektive als jeweils eine von vielfältigen Möglichkeiten des Ordnens und Deutens von Welt, um Kinder in der Erschliessung von komplexen Phänomenen ihrer Lebenswelt zu unterstützen $^{4}$ (GDSU 2013, 14). Die einzelnen Perspektiven beinhalten Erkenntnisse, Zugänge, Methoden und Arbeitsweisen der Fachkulturen, welche im Perspektivrahmen als inhaltliche Themenbereiche, Fragestellungen und Konzepte sowie prozedurale Denk-, Arbeits- und Handlungsweisen grundlegend formuliert wurden (GDSU 2013, 12). Im Perspektivrahmen werden zudem die perspektivenübergreifenden Denk-, Arbeits- und Handlungsweisen erkennen/verstehen, eigenständig erarbeiten, evaluieren/reflektieren, kommunizieren/mit anderen zusammenarbeiten, den Sachen interessiert begegnen und umsetzen/handeln formuliert, die sich über alle Perspektiven erstrecken.

Zur Darstellung wurde das Kreismodell des Perspektivrahmens der AG Medien \& Digitalisierung der GDSU (ehemals Neue Medien (ICT) im Sachunterricht) gewählt, da dieses den perspektivenvernetzenden Themenbereich Medien in den Mittelpunkt der sachunterrichtlichen Auseinandersetzung stellt (Peschel 2016, 13).

4 Weitere Konzeptionen des Sachunterrichts u.a. bei Köhnlein 2012, Thomas 2013, Kahlert 2016 und Nießeler 2020. 


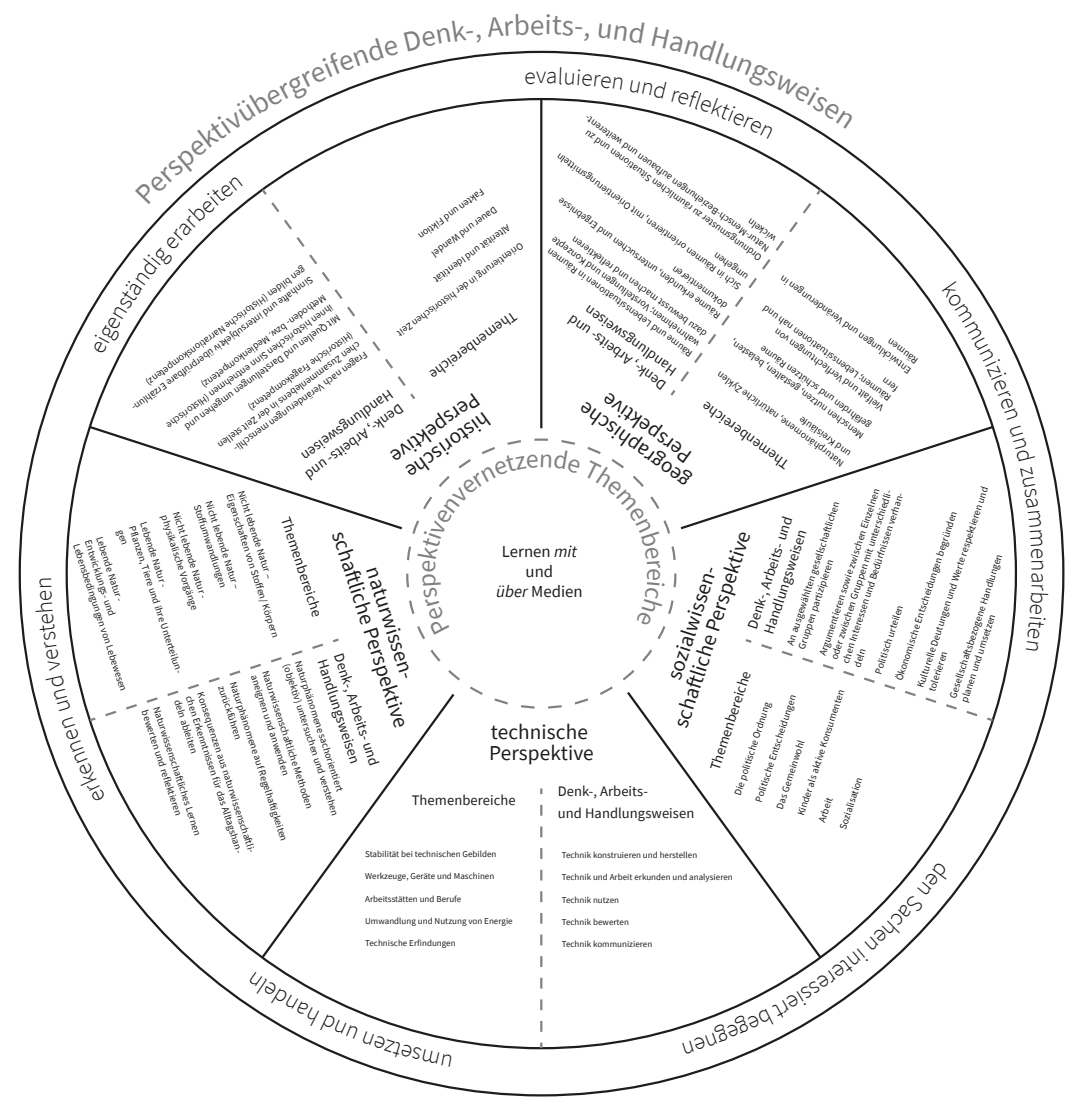

Abb. 1.: Kreismodell des Perspektivrahmens, erarbeitet von der AG Medien \& Digitalisierung der GDSU, Grafik Christian Borowski (Peschel 2016).

Die sozialwissenschaftliche Perspektive ermöglicht Grundschülerinnen und -schülern zum Beispiel in der Auseinandersetzung mit Politik, Wirtschaft, Recht, Kultur und Gemeinschaft am demokratischen Leben aktiv teilzunehmen, gesellschaftliche Probleme und Aufgaben zu erkennen, zu reflektieren und gegebenenfalls zu ihrer Lösung beizutragen (GDSU 2013, 27f.; Gläser und Richter 2015, 9). Der Perspektivrahmen beinhaltet die Themenbereiche: 1 . die politische Ordnung, 2. politische Entscheidungen, 3. das Gemeinwohl, 4. Kinder als aktive Konsumenten, 5. Arbeit und 6. Sozialisation (GDSU 2013, 34f.). Als perspektivenbezogene Denk-, Arbeits- und Handlungsweisen werden 1. an ausgewählten gesellschaftlichen Gruppen partizipieren, 2. argumentieren sowie zwischen Einzelnen oder zwischen Gruppen mit 
unterschiedlichen Interessen und Bedürfnissen verhandeln, 3. politisch urteilen, 4. ökonomische Entscheidungen begründen, 5. kulturelle Deutungen und Werte respektieren und tolerieren sowie 6. gesellschaftsbezogene Handlungen planen und umsetzen beschrieben (GDSU 2013, 30f.). Innerhalb der sozialwissenschaftlichen Perspektive wurden folgende Fragestellungen formuliert, die von den Seminarteilnehmenden als relevante Aspekte für das Lernen über digitale Medien erarbeitet wurden: Was sind soziale Netzwerke? Wie verhält man sich in sozialen Netzwerken? Wie kann man sich digital an Öffentlichkeit/Gruppen beteiligen? Wie kann man sich digital ausdrücken/ Öffentlichkeit herstellen? Wann ist der Kauf eines (neuen) digitalen Geräts sinnvoll? Welche Berufsgruppen entwickeln digitale Medien? Welche Berufe haben sich durch digitale Medien verändert, sind neu entstanden? Warum kauft man im Internet? Wie werden Daten gespeichert? Welche digitalen Medien sind für Kinder geeignet? Welche Regeln gelten bei der Nutzung von digitalen Medien, insbesondere an öffentlichen Orten? Welche Rechte haben Kinder im Internet? Wie kann man im Internet seine Privatsphäre schützen? Welche Vorbilder haben Kinder im Internet? Wie entstehen Werbeanzeigen im Internet? Um das Lernen mit digitalen Medien in die sozialwissenschaftlichen Denk-, Arbeits- und Handlungsweisen einzubeziehen, können Schülerinnen und Schüler eigene mediale Inhalte in Wikis produzieren und verantworten, fremde mediale Beiträge beurteilen und kommentieren sowie digitale Informationsseiten nutzen.

Die naturwissenschaftliche Perspektive ermöglicht Grundschülerinnen und -schülern in der Begegnung, Erschliessung und Auseinandersetzung mit belebter sowie unbelebter Natur, sich mit dem Verhältnis von Mensch und Natur auseinanderzusetzen, welches für die Gegenwart und Zukunft unter dem Aspekt der Nachhaltigkeit gestaltet werden soll (GDSU 2013, 37; Giest 2017, 7). Der Perspektivrahmen beschreibt die Themenbereiche: 1. Nicht lebende Natur- Eigenschaften von Stoffen/Körpern, 2. Nicht lebende Natur- Stoffumwandlungen, 3. Nicht lebende Natur- physikalische Vorgänge, 4. lebende Natur- Pflanzen, Tiere und ihre Unterteilungen sowie 5. lebende Natur- Entwicklungs- und Lebensbedingungen von Lebewesen. Die naturwissenschaftlichen Denk-, Arbeits- und Handlungsweisen umfassen 1. Naturphänomene sachorientiert (objektiv) untersuchen und verstehen, 2. naturwissenschaftliche Methoden aneignen und anwenden, 3. Naturphänomene 
auf Regelhaftigkeiten zurückführen, 4. Konsequenzen aus naturwissenschaftlichen Erkenntnissen für das Alltagshandeln ableiten und 5. naturwissenschaftliches Lernen bewerten und reflektieren. Innerhalb der naturwissenschaftlichen Perspektive wurden folgende Fragestellungen formuliert, die von den Studierenden für das Lernen über digitale Medien als relevant erachtet wurden: Aus welchen Materialien werden digitale Medien hergestellt? Wie und wo werden benötigte Stoffe abgebaut? Welche Eigenschaften haben diese Materialien? Wieviel Energie wird in einem Akku gespeichert? Welche Auswirkungen sind durch die zunehmende Digitalisierung in der Natur erkennbar? Um das Lernen mit digitalen Medien in die naturwissenschaftlichen Denk-, Arbeits- und Handlungsweisen einzubeziehen, können Schülerinnen und Schüler digitale Messerfassungen nutzen, Geräteteile von digitalen Medien digital gestützt auf Materialien und Eigenschaften untersuchen, Energieumsatz von Apps untersuchen und die Entladung des Akkus durch verschiedene Apps untersuchen.

Die geographische Perspektive ermöglicht Grundschülerinnen und -schülern, sich in ihrer natürlichen und hervorgebrachten Umwelt zu orientieren, die Unterschiedlichkeit von Räumen zu erfahren und zu erschliessen, wie unterschiedlich Menschen leben und ihre Umwelt nutzen (GDSU 2013, 46; Adamina, Hemmer, und Schubert 2016, 9f.). Ausgehend von ihren eigenen Erfahrungen beschäftigen sich Schülerinnen und Schüler exemplarisch mit für sie bedeutsamen Themen der Geographie, um Einblicke in das vielfältige Leben in der Welt sowie in lokale bis globale Beziehungen, Verflechtungen, Abhängigkeiten und Ungleichheiten zu erhalten (GDSU 2013, 46; Adamina, Hemmer, und Schubert 2016, 9f.). Der Perspektivrahmen beschreibt in der geographischen Perspektive die Themenbereiche: 1. Naturphänomene, natürliche Zyklen und Kreisläufe, 2. Menschen nutzen, gestalten, belasten, gefährden und schützen Räume, 3. Vielfalt und Verflechtungen von Räumen; Lebenssituationen nah und fern sowie 4. Entwicklungen und Veränderungen in Räumen (GDSU 2013, 51f.). Als perspektivenbezogene DenkArbeits- und Handlungsweisen werden 1. Räume und Lebenssituationen in Räumen wahrnehmen; Vorstellungen und Konzepte dazu bewusst machen und reflektieren, 2. Räume erkunden, untersuchen und Ergebnisse dokumentieren, 3. sich in Räumen orientieren, mit Orientierungsmitteln umgehen und 4. Ordnungsmuster zu räumlichen Situationen und zu Natur-Mensch-Beziehungen 
aufbauen und weiterentwickeln beschrieben (GDSU 2013, 48f.) Der Perspektivrahmen bezieht sich dabei in seinen Ausführungen auf (natürliche) Lebensräume (GDSU 2013, 46). Für die Erschliessung von digitalen Medien ist es daher notwendig, diese durch digitale/virtuelle Räume zu ergänzen. Innerhalb der geographischen Perspektive wurden folgende Fragestellungen formuliert, die von den Seminarteilnehmenden als relevant für das Lernen über digitale Medien erachtet wurden: Was sind digitale/virtuelle Räume? Wie unterscheiden sich digitale/virtuelle und natürliche Räume? Wie und wo haben sich durch die Entwicklung von digitalen Medien und den damit verbundenen Abbau von Rohstoffen Naturräume verändert? Wie kann man bei einem nachhaltigen Umgang mit digitalen Medien mitwirken? Wie unterstützen digitale Medien die Vorhersage von Naturereignissen? Um das Lernen mit digitalen Medien in die geographischen Denk-, Arbeits- und Handlungsweisen einzubeziehen, können Schülerinnen und Schüler digitale/virtuelle Räume erkunden, digitale/virtuelle Räume zur Information und Kommunikation nutzen, digitale (Geo-)Medien wie kidimaps/GPS/google streetview u.a. nutzen sowie Tätigkeiten in natürlichen und digitalen Räumen vergleichen (z.B. Fussball spielen, Arztsprechstunde, Einkauf, Arbeit).

Die historische Perspektive ermöglicht Grundschülerinnen und -schülern die Erfahrung, dass sich Vergangenheit, Gegenwart und Zukunft beeinflussen. Ausgehend von den eigenen Erfahrungen beschäftigen sich Schülerinnen und Schüler exemplarisch mit für sie bedeutsamen Themen im Wandel der Zeit, um die Fähigkeit zum historischen Denken und ein reflektiertes Geschichtsbewusstsein zu entwickeln (GDSU 2013, 56f.; Becher, Gläser, und Pleitner 2016, 9). In der historischen Perspektive ist die Bearbeitung und Beantwortung von geschichtlichen Fragen mithilfe von und durch die Auseinandersetzung mit Quellen und Darstellungen zentraler Gegenstand, um gegenwärtige Probleme und Phänomene besser zu verstehen und zukunftsbezogene Handlungsperspektiven zu entwickeln (GDSU 2013, 57). Der Perspektivrahmen beschreibt die Themenbereiche: 1. Orientierung in der historischen Zeit, 2. Alterität und Identität, 3. Dauer und Wandel sowie 4. Fakten und Fiktion (GDSU 2013, 6of.). Als perspektivenbezogene Denk- Arbeits- und Handlungsweisen werden 1. Fragen nach Veränderungen menschlichen Zusammenlebens in der Zeit stellen (Historische 
Fragekompetenz), 2. mit Quellen und Darstellungen umgehen und ihnen historischen Sinn entnehmen (historische Methoden- bzw. Medienkompetenz) sowie 3. sinnhafte und intersubjektiv überprüfbare Erzählungen bilden (Historische Narrationskompetenz) beschrieben (GDSU 2013, 58f.). Innerhalb der historischen Perspektive wurden folgende Fragestellungen formuliert, die für das Lernen über digitale Medien als relevant erachtet wurden: Wann und von wem wurden verschiedene digitale Medien wie Mobiltelefon, Smartphone, Computer, Tablet, Internet etc. erfunden? Gab es besondere Ereignisse, die zu den Erfindungen geführt haben? Wie hat sich das Leben der Menschen in Bezug auf Freizeitgestaltung, Alltag, Beruf, Kommunikation, Einkauf, zwischenmenschliche Beziehung etc. durch diese Medien geändert? Wie war es davor, wie war es zu Beginn der Entwicklungen, im Laufe der Zeit, heute und wie könnte es in naher und ferner Zukunft sein? Wie wurden Informationen und Daten vor der Entwicklung digitaler $\mathrm{Me}$ dien gespeichert und überliefert/weitergegeben und wie ist es heute? Um das Lernen mit digitalen Medien in die historischen Denk-, Arbeits- und Handlungsweisen einzubeziehen, können Schülerinnen und Schüler durch das Erstellen von digitalen Zeitleisten Erfindungen/Entwicklungen von digitalen Medien historisch einordnen, Befragungen von Zeitzeugen digital dokumentieren, frühere und heutige Methoden der Datenspeicherung durch die Verwendung verschiedener analoger und digitaler Medien wie Bücher/CDs/Clouds vergleichen, eigene historische Narrationen in Wikis produzieren und fremde Beiträge kritisch begutachten, in virtuellen Exkursionen historische Plätzen erkunden sowie digitale historische Quellen und Materialen wie Bilder/Texte/Videomaterial nutzen.

Die technische Perspektive ermöglicht Grundschülerinnen und -schülern Zugänge zu technischem Handeln und Denken, um Möglichkeiten und Folgewirkungen von Technik zu erkennen, eine humane und zukunftsfähige Technik mitzudenken, mit zu verantworten und mitgestalten zu können (GDSU 2013, 63). Dies beinhaltet das Erkennen zu Grunde liegender Funktionszusammenhänge von Technik und technischen Prinzipien, das Durchdringen von Funktionsweisen und Prozessen und die kritische Reflexion der Auswirkungen von Technik (GDSU 2013, 63). Der Perspektivrahmen beschreibt die Themenbereiche: 1. Stabilität bei technischen Gebilden, 2. Werkzeuge, Geräte und Maschinen, 3. Arbeitsstätten und Berufe, 4. Umwandlung 
und Nutzung von Energie und 5. technische Erfindungen (GDSU 2013, 68f.). Die technikbezogenen Denk-, Arbeits- und Handlungsweisen umfassen 1. Technik konstruieren und herstellen, 2. Technik und Arbeit erkunden und analysieren, 3. Technik nutzen, 4. Technik bewerten und 5. Technik kommunizieren (GDSU 2013, 65f.). Innerhalb der technischen Perspektive wurden folgende Fragestellungen von den Studierenden formuliert, die für das Lernen über digitale Medien als relevant erachtet wurden: Welche Bestandteile haben verschiedene digitale Medien? Wie sind die Bestandteile aufgebaut? Was ist Virtual/Augmented Reality? Wie werden Smartphones hergestellt? Wie entsorgt man digitale Geräte? Welche Gefahren (Strahlung, Abbau der Rohstoffe, Inhalte) und gesundheitliche Risiken (Sucht, Motion Sickness) können bei der Nutzung von digitalen Medien entstehen? Welchen Nutzen haben digitale Medien für Menschen? Wie werden digitale Medien in der Arbeitswelt, in der Schule und privat eingesetzt? Wieviel Energie benötigt eine App? Wie lange lädt ein Smartphone? Wie wurden digitale Medien bisher weiterentwickelt? Wie können verschiedene digitale Medien in Zukunft weiterentwickelt werden? Wie werden digitale Daten gespeichert? Um das Lernen mit digitalen Medien in die technischen Denk-, Arbeitsund Handlungsweisen einzubeziehen, können Schülerinnen und Schüler Microcomputer programmieren, verschiedene digitale Medien untersuchen und digital gestützt vergleichen sowie eigene App-Ideen entwickeln und umsetzen.

\section{Fazit}

Aufgrund der Komplexität von digitalen Medien ist eine Aneinanderreihung von einzelnen Aspekten der verschiedenen Perspektiven im Sinne einer «Perspektivenaddition» nicht ausreichend (Schmid u. a. 2013, 49). Die dargestellten Aspekte müssen miteinander vernetzt und in gegenseitiger Abhängigkeit erschlossen werden, um relevante Sachzusammenhänge und Wechselwirkungen zu verstehen (Köhnlein 2011, 16; Gesellschaft für Informatik 2016, 2; AG Medien \& Digitalisierung der GDSU 2019). Hierfür eignet sich die Entwicklung einer übergeordneten Fragestellung als vernetzendes und verbindendes Element (Pech 2009; Schmid u. a. 2013). Die übergeordnete Fragestellung dient als Schlüssel (Schmid u. a. 2013, 49) für 
ein «Zusammenheitsdenken» und erfordert in der Entwicklung den Einbezug mehrerer Aspekte: Die Fragestellung muss anschlussfähig an die Lebenswelt der Grundschülerinnen und -schüler sein und gegenwärtig und zukünftig von Bedeutung sein. Die exemplarische Erkenntnisse sollen auf andere Situationen übertragbar sein, Wissensbestände verschiedener Perspektiven aufgreifen und miteinander in Verbindung setzen, komplex formuliert und Spannungsfelder beinhalten (Schmid u. a. 2013, 49). Die Konkretisierung von Inhalten sowie Denk-, Arbeits- und Handlungsweisen innerhalb der verschiedenen Perspektiven ist als (vorläufiger) Überblick in Bezug auf das Lernen mit und über digitale Medien in der grundlegenden Struktur des Perspektivrahmens zu verstehen. Er gibt erste Hinweise darauf, welche Aspekte für die Erschliessung von digitalen Medien im Sachunterricht relevant sein können. Es ist sinnvoll, diese zu prüfen, weiter auszudifferenzieren und übergeordnete Fragestellungen zu entwickeln.

\section{Literatur}

Adamina, Marco, Michael Hemmer, und Jan Christoph Schubert, Hrsg. 2016. Die geographische Perspektive konkret. Begleitband 3 zum Perspektivrahmen Sachunterricht. Bad Heilbrunn: Verlag Julius Klinkhardt.

AG Medien \& Digitalisierung der GDSU. 2019. "Sachunterricht und Digitalisierung. Positionspapier». https://www.researchgate.net/publication/336899363_Sachunterricht_und_Digitalisierung.

Albers, Stine. 2017. «Bildung und Vielperspektivität im Sachunterricht - ein 〈inniges〉 Verhältnis». GDSU-Journal, Nr. 6: 11-20.

Becher, Andrea, Eva Gläser, und Berit Pleitner, Hrsg. 2016. Die historische Perspektive konkret. Begleitband 2 zum Perspektivrahmen Sachunterricht. Bad Heilbrunn: Verlag Julius Klinkhardt.

Behrens, Julia, Ulrich Schmid, Lutz Goertz, und Julia Behrens. 2017. «Die Schulen im digitalen Zeitalter». Bertelsmann-Stiftung.

CARLSEN Verlag. 2019. «LeYo!» 21. Dezember 2019. https://www.carlsen.de/serie/ leyo/58215.

Davis, Niki, Birgit Eickelmann, und Pinelopi Zaka. 2013. «Restructuring of educational systems in the digital age from a co-evolutionary perspective», Journal of Computer-Assisted Learning, 29 (5): 438-50. https://doi.org/10.1111/ jcal.12032.

Döbeli Honegger, Beat. 2007. «Digital Naives». Beats Biblionetz - Begriffe (blog). 18. November 2007. http://beat.doebe.li/bibliothek/w02038.html. 
Döbeli Honegger, Beat. 2016. Mehr als O und 1: Schule in einer digitalisierten Welt. 1. Aufl. Bern: hep, der Bildungsverlag.

Drossel, Kerstin, Birgit Eickelmann, Heike Schaumburg, und Amelie Labusch. 2019. "Kapitel VII. Nutzung digitaler Medien und Prädiktoren aus der Perspektive der Lehrerinnen und Lehrer im internationalen Vergleich». In ICILS 2018 \#Deutschland Computer- und informationsbezogene Kompetenzen von Schülerinnen und Schülern im zweiten internationalen Vergleich und Kompetenzen im Bereich Computational Thinking., herausgegeben von Birgit Eickelmann, Wilfried Bos, Julia Gerick, Frank Goldhammer, Heike Schaumburg, Knut Schwippert, Martin Senkbeil, und Jan Vahrenhold, 205-40. Münster: Waxmann Verlag GmbH. https://kw.uni-paderborn.de/fileadmin/fakultaet/ Institute/erziehungswissenschaft/Schulpaedagogik/ICILS_2018__Deutschland_Berichtsband.pdf.

Eickelmann, Birgit. 2016. «Eine Bilanz zur Integration digitaler Medien an Grundschulen in Deutschland aus international vergleichender Perspektive». In Neue Medien in der Grundschule 2.0. Grundlagen - Konzepte - Perspektiven., herausgegeben von Markus Peschel und Thomas Irion, 79-90. Beiträge zur Reform der Grundschule. 141. Frankfurt am Main: Grundschulverband e.V.

Eickelmann, Birgit, Wilfried Bos, und Amelie Labusch. 2019. «Kapitel I. Die Studie ICILS 2018 im Überblick - Zentrale Ergebnisse und mögliche Entwicklungsperspektiven». In ICILS 2018 \#Deutschland Computer- und informationsbezogene Kompetenzen von Schülerinnen und Schülern im zweiten internationalen Vergleich und Kompetenzen im Bereich Computational Thinking., herausgegeben von Birgit Eickelmann, Wilfried Bos, Julia Gerick, Frank Goldhammer, Heike Schaumburg, Knut Schwippert, Martin Senkbeil, und Jan Vahrenhold, 7-31. Münster: Waxmann Verlag GmbH. https://kw.uni-paderborn.de/fileadmin/ fakultaet/Institute/erziehungswissenschaft/Schulpaedagogik/ICILS_2018__ Deutschland_Berichtsband.pdf.

GDSU, Hrsg. 2013. Perspektivrahmen Sachunterricht. Vollständig überarbeitete und Erweiterte Ausgabe. Bad Heilbrunn: Verlag Julius Klinkhardt.

Gervé, Friedrich. 2015. «Digitale Medien». In Handbuch Didaktik des Sachunterrichts. 2., aktualisierte und erw. Aufl., herausgegeben von Joachim Kahlert, Maria Fölling-Albers, Margarete Götz, Andreas Hartinger, Susanne Miller, und Steffen Wittkowske, 496-500. UTB. 8621. Bad Heilbrunn: Klinkhardt.

Gervé, Friedrich. 2016. «Digitale Medien als "Sache" des Sachunterrichts». In Neue Medien in der Grundschule 2.o. Grundlagen - Konzepte - Perspektiven., herausgegeben von Markus Peschel und Thomas Irion, 121-34. Beiträge zur Reform der Grundschule. 141. Frankfurt am Main: Grundschulverband e.V.

Gervé, Friedrich. 2019. «Digitalisierung und Bildung in der Grundschule». In Digitale Transformation in der Bildungslandschaft - den analogen Stecker ziehen?, herausgegeben von Jacqueline Heider-Lang und Alexandra Merkert, 1. Augsburg, München 2019, 98-114. Mering: Rainer Hampp Verlag. 
Gesellschaft für Fachdidaktik. 2018. «Fachliche Bildung in der digitalen Welt. Positionspapier der Gesellschaft für Fachdidaktik». http://www.fachdidaktik. org/wp-content/uploads/2018/07/GFD-Positionspapier-Fachliche-Bildung-inder-digitalen-Welt-2018-FINAL-HP-Version.pdf.

Gesellschaft für Informatik. 2016. «Dagstuhl-Erklärung. Bildung in der digitalen vernetzten Welt. Eine gemeinsame Erklärung der Teilnehmerinnen und Teilnehmer des Seminars auf Schloss Dagstuhl - Leibniz-Zentrum für Informatik GmbH». https://gi.de/fileadmin/GI/Hauptseite/Themen/Dagstuhl-Erkla__rung_2016-03-23.pdf.

Gesellschaft für Informatik. 2019. «Kompetenzen für informatische Bildung im Primarbereich», Beilage zu LOG IN 39 (191/192). https://dl.gi.de/bitstream/ handle/20.500.12116/20121/61-GI-Empfehlung_Kompetenzen_informatische_ Bildung_Primarbereich.pdf.

Gesellschaft für Medienpädagogik und Kommunikationskultur. 2019. «Stellungnahme der GMK zum „DigitalPakt Schule” Ein großer Schritt für die technische Infrastruktur, ein zu kleiner für die schulische Medienbildung». https://www.gmk-net.de/2019/04/30/stellungnahme-der-gmk-zum-digitalpaktschule/.

Gewerkschaft Erziehung und Wissenschaft. 2019. «Empfehlungen zur Umsetzung des Digitalpakts». https://www.gew.de/fileadmin/media/sonstige_ downloads/hv/Digitale-Medienbildung/2019-04-30_Empfehlungen_zur_Umsetzung_des_Digitalpakts.pdf.

Giest, Hartmut, Hrsg. 2017. Die naturwissenschaftliche Perspektive konkret. Begleitband 4 zum Perspektivrahmen Sachunterricht. Bad Heilbrunn: Verlag Julius Klinkhardt.

Giest, Hartmut, Andreas Hartinger, und Sandra Tänzer. 2017. «Editorial». In Vielperspektivität im Sachunterricht, herausgegeben von Hartmut Giest, Andreas Hartinger, und Sandra Tänzer, 9-12. Probleme und Perspektiven des Sachunterrichts. 27. Bad Heilbrunn: Verlag Julius Klinkhardt.

Gläser, Eva, und Dagmar Richter, Hrsg. 2015. Die sozialwissenschaftliche Perspektive konkret. Begleitband I zum Perspektivrahmen Sachunterricht. Bad Heilbrunn: Verlag Julius Klinkhardt.

Grundschulverband. 2015. «Standpunkt Medienbildung. Grundschulkinder bei der Mediennutzung begleiten - Innovative Lernpotenziale in der Grundschule nutzen». Grundschule aktuell, Nr. 131: 20-21.

Grundschulverband. 2018. «Digitale Mündigkeit beginnt in der Grundschule! Stellungnahme des Grundschulverbands zum "DigitalPakt Schule" und zum KMK-Beschluss „Bildung in der digitalen Welt"». https://grundschulverband. de/wp-content/uploads/2018/08/stellungnahme-gsv-digitalpakt-schule.pdf.

Irion, Thomas. 2018. «Wozu digitale Medien in der Grundschule? Sollte das Thema Digitalisierung in der Grundschule tabuisiert werden?» Grundschule aktuell, Nr. 142: 3-7. 
Irion, Thomas, und Markus Peschel. 2016. «Grundschule und neue Medien - Neue Entwicklungen». In Neue Medien in der Grundschule 2.0. Grundlagen - Konzepte - Perspektiven., herausgegeben von Markus Peschel und Thomas Irion, 11-15. Beiträge zur Reform der Grundschule. 14l. Frankfurt am Main: Grundschulverband e.V.

Kahlert, Joachim, Hrsg. 2016. Der Sachunterricht und seine Didaktik. 4., aktualisierte Auflage. Bad Heilbrunn: Verlag Julius Klinkhardt.

Klafki, Wolfgang. 1992. «Allgemeinbildung in der Grundschule und der Bildungsauftrag des Sach- unterrichts». In Brennpunkte des Sachunterrichts. Vorträge zur Gründungstagung der Gesellschaft für Didaktik des Sachunterrichts e. V. vom 19. bis 21. März 1992 in Berlin, herausgegeben von Roland Lauterbach, Walter Köhnlein, Kay Spreckelsen, und Elard Klewitz, 11-31. Kiel: pedocs. http://nbnresolving.de/urn:nbn:de:0111-pedocs-129098.

Köhnlein, Walter. 2011. «Die Bildungsaufgaben des Sachunterrichts und der genetische Zugriff auf die Welt», 14.

Köhnlein. 2012. Sachunterricht und Bildung. Bad Heilbrunn: Klinkhardt.

Köhnlein, Walter, Brunhilde Marquardt-Mau, und Ludwig Duncker. 2013. «Vielperspektivität». www.widerstreit-sachunterricht.de, Nr. 19: 1-3.

Kultusministerkonferenz. 2012. «Medienbildung in der Schule». https://www.kmk. org/fileadmin/Dateien/veroeffentlichungen_beschluesse/2012/2012_03_08_ Medienbildung.pdf.

Kultusministerkonferenz. 2016. «Bildung in der digitalen Welt. Strategie der Kultusministerkonferenz». https://www.kmk.org/fileadmin/Dateien/pdf/PresseUndAktuelles/2018/Digitalstrategie_2017_mit_Weiterbildung.pdf.

Lauterbach, Roland. 2017. «Vielperspektivität-ein Beitrag zur Identitätsfindung der Didaktik des Sachunterrichts». In Vielperspektivität im Sachunterricht, herausgegeben von Hartmut Giest, Andreas Hartinger, und Sandra Tänzer, 13-26. Probleme und Perspektiven des Sachunterrichts. 27. Bad Heilbrunn: Verlag Julius Klinkhardt.

LEGO. 2018. «LEGO ${ }^{\circledR}$ Playgrounds - first ever portal to discover and play with the latest LEGO mixed reality experiments». 2018. https://www.lego.com/dede/aboutus/news/2019/october/lego-playgrounds/.

Lenkungskreis der Initiative KBoM. 2019. «Das Medienpädagogische Manifest Addendum 2019». 2019. https://www.keine-bildung-ohne-medien.de/wp-content/uploads/2019/og/Medienp\%C3\%A4dagogischesManifestAddendum2019. pdf.

Medienpädagogischer Forschungsverbund Südwest. 2018. "KIM-Studie 2018. Kindheit, Internet, Medien. Basisuntersuchung zum Medienumgang 6- bis 13-Jähriger KIM Studie 2018». https://www.mpfs.de/fileadmin/files/Studien/ KIM/2018/KIM-Studie_2018_web.pdf.

Medienpädagogischer Forschungsverbund Südwest. 2019. "Pressemitteilung. Jedes dritte Kind nutzt täglich WhatsApp. KIM-Studie 2018 zum Medienumgang 6- bis 13-Jähriger veröffentlicht». https://www.mpfs.de/fileadmin/files/ Presse/2019/PM_1_2019_VOEKIM18.pdf. 
Mitzlaff, Hartmut. 2016. «Medien inklusive - inklusive Mediendidaktik und Medienpädagogik des Sachunterrichts». In Mediales Lernen. Beispiele für eine inklusive Mediendidaktik., herausgegeben von Markus Peschel, 7:17-34. Dimensionen des Sachunterrichts. Baltmannsweiler: Schneider Verlag Hohengehren $\mathrm{GmbH}$.

Mitzlaff, Hartmut, und Angelika Speck-Hamdan. 1998. "Grundschule und neue Medien». In Beiträge zur Reform der Grundschule. 103. Grundschule und neue Medien, 10-34. Frankfurt, Main: Arbeitskreis Grundschule.

Moser, Heinz. 2019. Einführung in die Medienpädagogik. Aufwachsen im digitalen Zeitalter. 6. überarbeitete und aktualisierte Auflage. Lehrbuch. Wiesbaden: Springer VS.

Pech, Detlef. 2009. «Sachunterricht - Didaktik und Disziplin. Annäherungen an ein Sachlernverständnis im Kontext der Fachentwicklung des Sachunterrichts und seiner Didaktik». www.widerstreit-sachunterricht.de, Nr. 13: 1-10.

Peschel, Markus. 2016. «Mediales Lernen - Eine Modellierung als Einleitung». In Mediales Lernen. Beispiele für eine inklusive Mediendidaktik., herausgegeben von Markus Peschel, 7:7-16. Dimensionen des Sachunterrichts. Baltmannsweiler: Schneider Verlag Hohengehren GmbH.

Peschel, Markus. 2019a. "Arme Kinder - arme Schule. Wie gerecht ist unser Bildungssystem?», Grundschule aktuell, 148: 26-29.

Peschel, Markus. 2019b. «Milliarden für die Bildung. Grundlegende Forderungen an den Digitalpakt», Die GRUNDSCHULZEITSCHRIFT, 318: 43.

Reiss, Kristina, Mirjam Weis, Eckhard Klieme, und Olaf Köller. 2019. PISA 2018 Grundbildung im internationalen Vergleich. Zusammenfassung. Waxmann Verlag.

Schaumburg, Heike, Julia Gerick, Birgit Eickelmann, und Amelie Labusch. 2019. «Kapitel VIII. Nutzung digitaler Medien aus der Perspektive der Schülerinnen und Schüler im internationalen Vergleich». In ICILS 2018 \#Deutschland Computer- und informationsbezogene Kompetenzen von Schülerinnen und Schülern im zweiten internationalen Vergleich und Kompetenzen im Bereich Computational Thinking., herausgegeben von Birgit Eickelmann, Wilfried Bos, Julia Gerick, Frank Goldhammer, Heike Schaumburg, Knut Schwippert, Martin Senkbeil, und Jan Vahrenhold, 241-70. Münster: Waxmann Verlag GmbH. https:// kw.uni-paderborn.de/fileadmin/fakultaet/Institute/erziehungswissenschaft/ Schulpaedagogik/ICILS_2018__Deutschland_Berichtsband.pdf.

Schmid, Kuno, Paolo Trevisan, Christine Künzli David, und Antonietta Di Giulio. 2013. «Die übergeordnete Fragestellung als zentrales Element im Sachunterricht». In SaCHen unterriCHten. Beiträge zur Situation der Sachunterrichtsdidaktik in der deutschsprachigen Schweiz, herausgegeben von Markus Peschel, Pascal Favre, und Christian Mathis, 41-54. https://forschdb2.unibas.ch/inf2/ rm_projects/object_view.php?r=2839365.

Schorb, Bernd, Anja Hartung-Griemberg, und Christine Dallmann, Hrsg. 2017. «Handlungsorientierte Medienpädagogik». In Grundbegriffe Medienpädagogik, 6., neu verfasste Auflage, 134-40. München: kopaed. 
Sektion Medienpädagogik, DGfE. 2016. «Stellungnahme zum Entwurf einer Strategie der Kultusministerkonferenz "Bildung in der digitalen Welt" Handlungsfelder (1) (2) und (4)». https://www.dgfe.de/fileadmin/OrdnerRedakteure/ Sektionen/Sek12_MedPaed/2016_Sektion_Medienpa\%CC\%88dagogik_der_ DGfE_zur_KMK-Strategie.pdf.

Spielwarenmesse. 2019. «Elektronisches Spielzeug». Spielwarenmesse Nürnberg. 2019. https://www.spielwarenmesse.de/messe/produkte-hallen/elektronisches-spielzeug/.

Statistisches Bundesamt. 2019. "Allgemeinbildende und berufliche Schulen». 2019. https://www.destatis.de/DE/Themen/Gesellschaft-Umwelt/BildungForschung-Kultur/Schulen/Tabellen/allgemeinbildende-beruflicheschulenschularten.html.

Straube, Philipp, Martin Brämer, Hilde Köster, und Ralf Romeike. 2018. «Eine digitale Perspektive für den Sachunterricht? Fachdidaktische Überlegungen und Implikationen». www.widerstreit-sachunterricht.de, Nr. 24: 1-11. http://www. widerstreit-sachunterricht.de/ebeneI/superworte/zumsach/straubeetal.pdf

Thom, Sabrina, Julia Behrens, Ulrich Schmid, und Lutz Goertz. 2017. «Digitales Lernen an Grundschulen». Bertelsmann-Stiftung. https://www.bertelsmannstiftung.de/fileadmin/files/BSt/Publikationen/GrauePublikationen/BSt_ MDB3_Schulen_web.pdf.

Thomas, Peter. 2019. «Messe-Neuheiten 2019: Elektronisches Spielzeug». 2019. https://www.spielwarenmesse.de/magazin/artikeldetail/elektronisches-spielzeug-neu-2019/.

Tulodziecki, Gerhard, Silke Grafe, und Bardo Herzig. 2019. Medienbildung in Schule und Unterricht: Grundlagen und Beispiele. 2. vollständig überarbeitete und aktualisierte Auflage. Bad Heilbrunn: Verlag Julius Klinkhardt. 